Supplement of Foss. Rec., 24, 275-285, 2021

https://doi.org/10.5194/fr-24-275-2021-supplement

(C) Author(s) 2021. CC BY 4.0 License.

(c) (1)

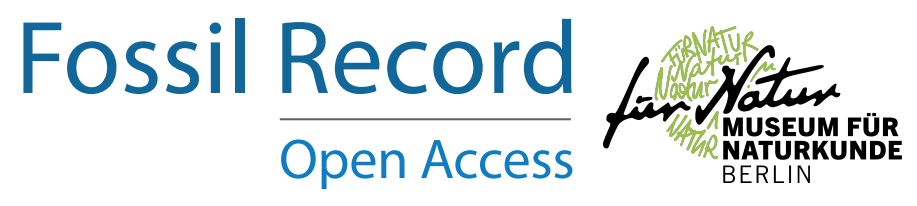

Supplement of

\title{
A new odontocete (Inioidea, Odontoceti) from the late Neogene of North Carolina, USA
}

Stephen J. Godfrey et al.

Correspondence to: Stephen J. Godfrey (stephen.godfrey@calvertcountymd.gov)

The copyright of individual parts of the supplement might differ from the article licence. 


\section{SUPPLEMENTARY PHYLOGENETIC INFORMATION}

We attempted to determine the phylogenetic position of Isoninia borealis by performing multiple phylogenetic analyses under differing protocols, using different matrices from the primary literature. We first coded I. borealis for the Pyenson et al. (2015) matrix, which consisted of 311 characters and 53 taxa. This matrix was then imported into TNT 1.5 (Goloboff $\&$ Catalano, 2016). All characters were unordered and we obtained our most parsimonious trees by using the "New Technology Search" criterion, using "sect. search", "ratchet", "drift", and "tree fusing" options all selected, and the "stabilize consensus" option selected for a total of 20 times. This was then followed by two rounds of tree bisection-reconnection (TBR) on the trees obtained by the new search criterion. Clade support was then calculated for the most parsimonious tree in TNT 1.5 using a script for Bremer Support, and Bootstrap Support was calculated with 1,000 replicates.

We then used the matrix from Lambert et al. (2020) to determine the phylogenetic affinity of $I$. borealis, which consisted of 324 characters and 110 taxa, which was also analyzed in PAUP*4.0a169. All 324 characters were weighted and 146 characters were ordered. This analysis also included a molecular constraint tree from the most updated genomic data from extant cetaceans (McGowen et al., 2020; Lambert et al., 2020). This molecular constraint tree was set as the backbone for which the heuristic search algorithm for the most parsimonious trees was performed. For this analysis, we ran the matrix under both the ACTRAN and DELTRAN parsimony setting for the identification of unambiguous synapomorphies uniting the clades of interest. Clades with branch lengths equal to zero were collapsed in accordance with Coddington and Scharff's fourth rule (Coddington \& Scharff, 1994; Kearney \& Clark, 2003), along with setting the step matrix to observable traits only, so that clades could not be defined by characters coded as "?" for any of the ingroup taxon. The outgroup was defined by 3 taxa: Bos taurus, Hippopotamus amphibius, and Sus scrofa. A traditional heuristic search was implemented with 900,000 replicates using TBR branch swapping algorithm. This was then followed by another round of TBR to ensure the most parsimonious trees were likely found.

We then replicated the Lambert et al. (2020) analyses protocol for elimination of wildcard taxa, and deleted (Auroracetus bakerae, Ischyrorhnychus vanbenedeni, Isthminia panamensis, 
Kwanzacetus khoisani, Lophocetus calvertensis, Pithanodelphis cornutus, Protophocaena minima, Scaldiporia vandokkumi, and Stenasodelphis russellae) as per their analysis for determining the phylogenetic affinity of Samaydelphis chacltanae. For Lambert et al. (2020), wildcard taxa were termed as taxa missing a significant portion of the character codings, especially in the otic region (Lambert et al., 2020). I. borealis was then analyzed in this reduced dataset (101 taxa); however, it must be stated that given the rigid protocols of Lambert et al. (2020), I. borealis does not meet the criterion for number of codeable characters, as the otic region is absent in the holotype specimen and this would likely have also led to the elimination of $I$. borealis from their analysis. The reduced taxon matrix was then reanalyzed following the same settings as the previous analysis as listed above for the Lambert et al. (2020) data. Clade support was also calculated in PAUP*4.0a169 in the form of Bootstrap support with 1,000 replicates. Bremer support for a similar analysis (Lambert et al., 2017) was calculated in TNT, and reported by shifting the decimal place two units to the left to account for the character weighting scheme employed. Since our analysis was based on a PAUP* based formatted dataset, we would have to manually calculate only the whole unit increases in Bremer support (e.g. 1, 2, $3,4,5,6,7)$ rather than using a script to calculate Bremer Support with decimal values. The validity and interpretability of Bremer Support for this analysis is questionable given that the topology was constrained to fit the molecular evidence, and the weighting scheme and ordering of the characters. We therefore chose to rely on Bootstrap support values over those of Bremer support in the evaluation of clade robustness.

\section{Results}

The results of all of these analyses result in conflicting trees, with the position of $I$. borealis labile to the matrix and taxa utilized in the analysis, as illustrated by Figures 1,2, and 3 . With the Pyenson et al. (2015) matrix (Figure 1), we find support for I. borealis as a derived inioid species with a sister taxon relationship to Isthminia panamensis. Interestingly, from this analysis, I. borealis is more closely related to Inia geoffrensis than to Meherrinia isoni, which is from the late Miocene marine deposits of the Eastover Formation in North Carolina, U.S.A. (Geisler et al., 2012), and is likely stratigraphically congruent with I. borealis. The results from the Lambert et al. (2020) analysis of the complete dataset placed I. borealis outside of the iniod clade, as seen in Figure 2. However, once the nine wildcard/incomplete taxa, including four 
fossil iniod taxa were removed from the analysis, and the dataset was then reran under the same parameters, I. borealis was recovered as a member of the iniod clade and as a sister taxon to Meherrinia isoni, with relatively strong Bootstrap support of $70 \%$. The two taxa formed a subclade within Inioidea that included Samaydelphis chacaltanae, and this clade was strongly supported with a Bootstrap value of $89 \%$.

We suggest further revision of the character matrices used for odontocete phylogeny, given the large amount of homoplasy within the data, and the continued search for more complete specimens of fragmentary taxa. Methods should be allocated to extant taxa to identify modules within the skeletal morphology of these organisms, so that integrated characters are not being oversampled in the character matrices. Results from these new character matrices should then be compared to the evidence from molecular studies in order to assess the fidelity of the morphological data accurately reconstructing the evolutionary history of these organisms. 


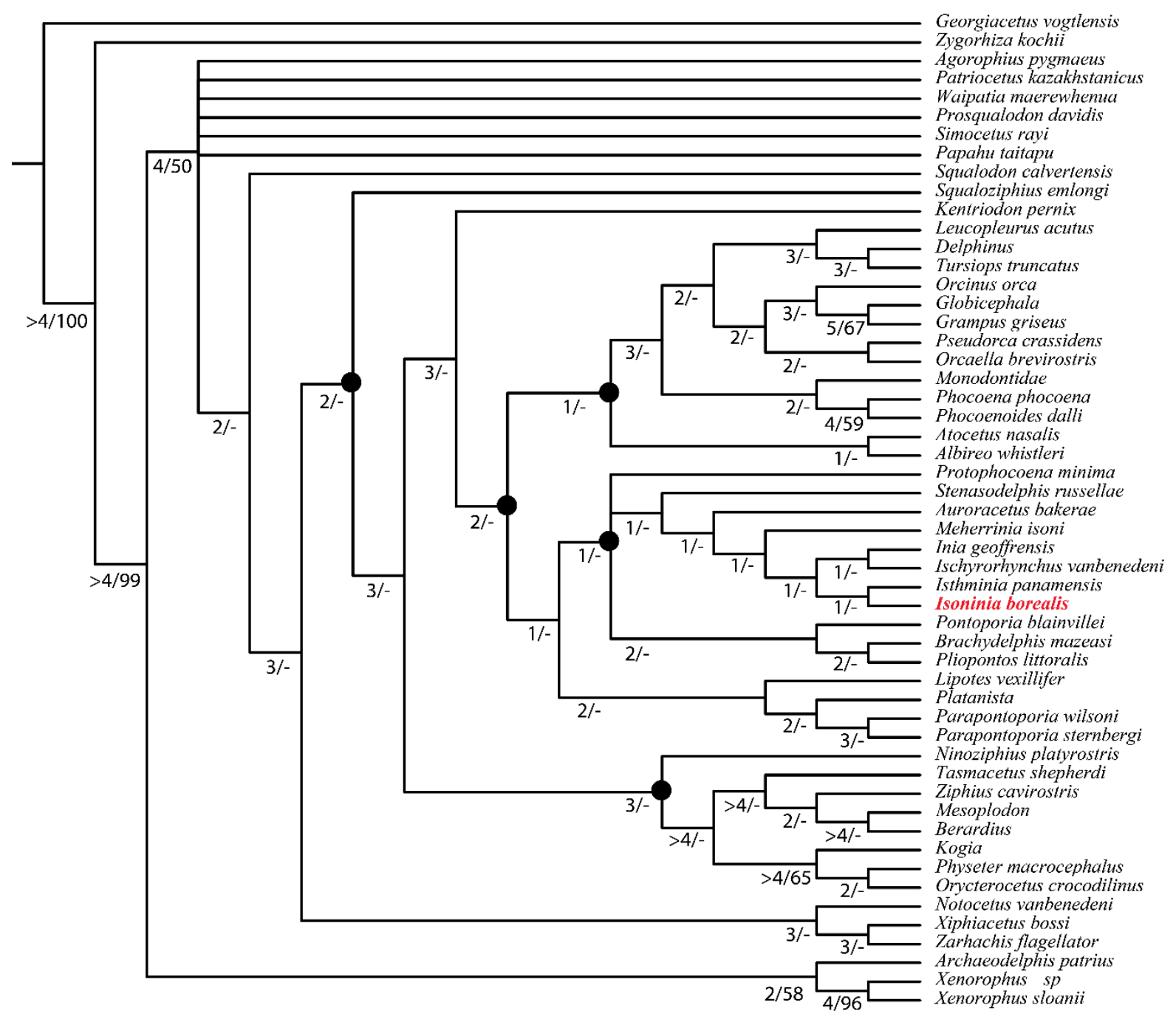

Figure 1. Strict consensus tree recovered using the Pyenson et al. (2015) matrix with a total of 53 taxa to determine the phylogenetic position of Isoninia borealis (highlighted in red font). This analysis recovered a total of 7 trees were obtained with a tree length of 1,928 (Consistency Index $(\mathrm{CI})=0.279$, Retention Index $(\mathrm{RI})=0.451)$. Bremer Support and Bootstrap values are reported at the nodes (Bremer Support/Bootstrap Support). Nodes that feature Bootstrap Support less than $50 \%$ are reported as (-) following the Bremer Support value. This analysis supported a sister taxon relationship between I. borealis and Isthminia panamensis. Abbreviations: D, Delphinida; DE, Delphinoidea; I, Inioidea; Z+Ph, Ziphiidae and Physeteroidea. 


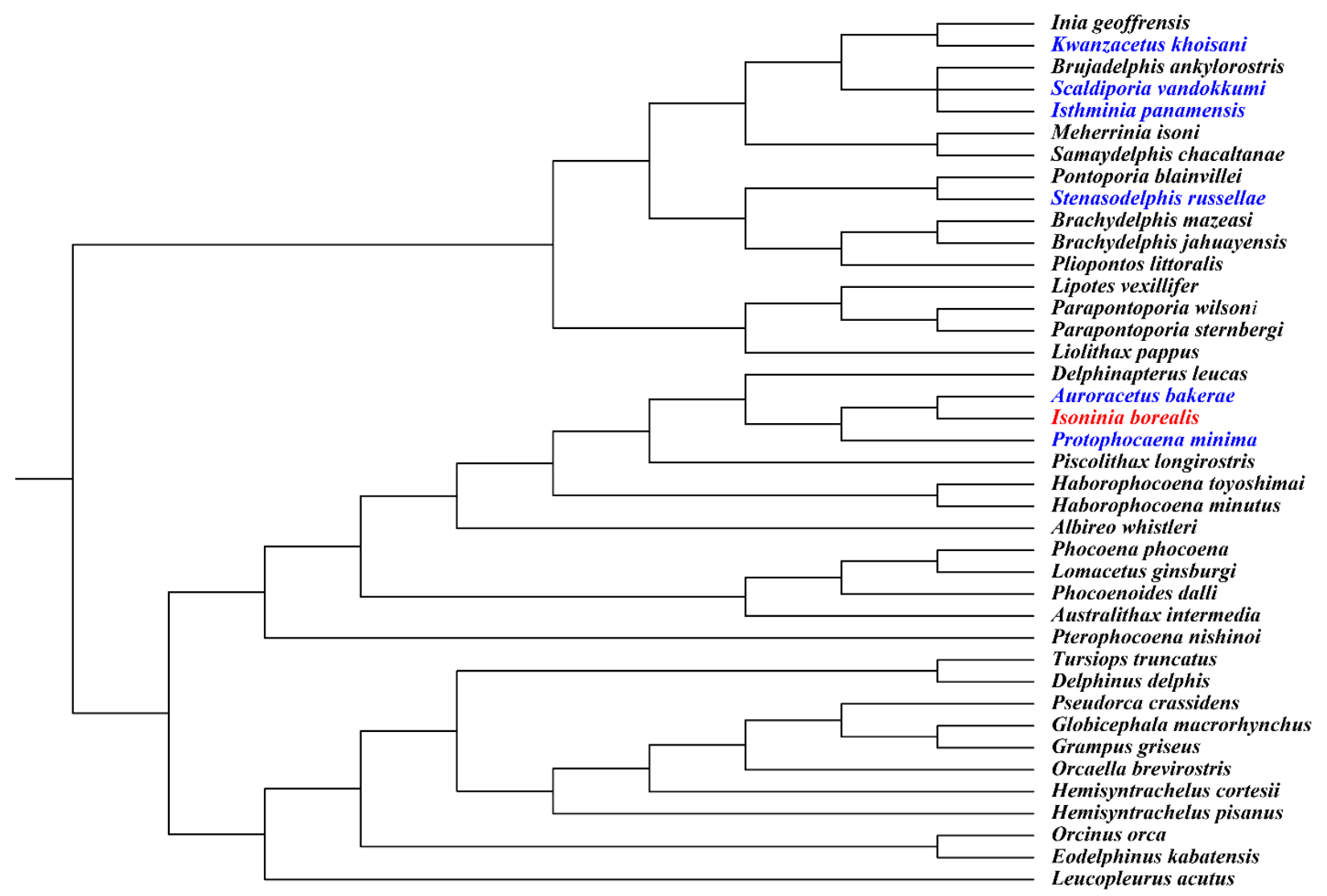

Figure 2. Strict consensus tree from the complete dataset from Lambert et al (2020) of 110 taxa for determination of the phylogenetic position of Isoninia borealis (highlighted in red font). I. borealis was recovered outside the clade Inioidea. Taxa that were then eliminated from the final analysis are highlighted in a blue font, and include four fossil iniods. A total of 2 most parsimonious trees were recovered from this analysis, with a tree length of $2319.77, \mathrm{CI}=0.157$, and $\mathrm{RI}=0.554$.

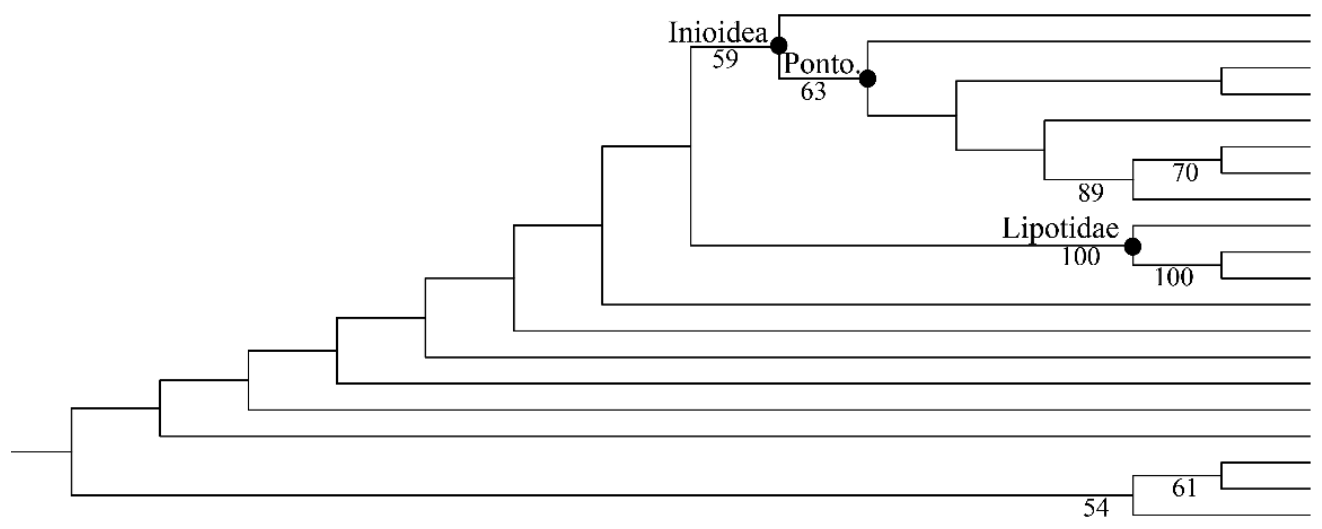

Inia geoffrensis Pontoporia blainvillei Brachydelphis mazeas Brachydelphis jahuayensis Pliopontos littoralis Meherrinia isoni Isoninia borealis Samaydelphis chacaltanae Lipotes vexillifer Parapontoporia wilsoni Parapontoporia sternbergi Brujadelphis ankylorostris Lophocetus repenningi Atocetus iquensis Atocetus nasalis Liolithax pappus Tagicetus joneti Kampholophus serrulus Macrokentriodon morani Hadrodelphis calvertense 
Figure 3. Strict consensus tree from the reduced taxonomic dataset (101 taxa) from Lambert et al (2020) focused in on the Inioidea clade, with special interest on the phylogenetic position of Isoninia borealis (highlighted in red font). The numbers along each of the branches are the Bootstrap support values for the subsequent clades. Clades that do not feature a number, feature Bootstrap support less than 50. There was only a single tree recovered, with a tree length of 2251.27, $\mathrm{CI}=0.1613$, and $\mathrm{RI}=0.5546$. From this analysis $I$. borealis was recovered within the clade Inoidea, and a sister taxon to Meherrinia isonii with relatively high Bootstrap support (70\%). Abbreviations: Ponto, Pontoporoiidae.

\section{Literature Cited}

Kearney, M., \& Clark, J. M. (2003). Problems due to missing data in phylogenetic analyses including fossils: a critical review. Journal of Vertebrate Paleontology, 23(2), 263-274.

Coddington, J., and N. Scharff. 1994. Problems with zero-lengthbranches. Cladistics 10:415-424.

Geisler, J. H., Godfrey, S. J., \& Lambert, O. (2012). A new genus and species of late Miocene inioid (Cetacea, Odontoceti) from the Meherrin River, North Carolina, USA. Journal of Vertebrate Paleontology, 32(1), 198-211.

Goloboff, P. A., \& Catalano, S. A. (2016). TNT version 1.5, including a full implementation of phylogenetic morphometrics. Cladistics, 32(3), 221-238.

Lambert, O., Bianucci, G., Urbina, M., \& Geisler, J. H. (2017). A new inioid (Cetacea, Odontoceti, Delphinida) from the Miocene of Peru and the origin of modern dolphin and porpoise families. Zoological Journal of the Linnean Society, 179(4), 919-946.

Lambert, O., Collareta, A., Benites-Palomino, A., Di Celma, C., De Muizon, C., Urbina, M., \& Bianucci, G. (2020). A new small, mesorostrine inioid (Cetacea, Odontoceti, Delphinida) from four upper Miocene localities in the Pisco Basin, Peru. Papers in Palaeontology.

McGowen, M. R., Tsagkogeorga, G., Álvarez-Carretero, S., Dos Reis, M., Struebig, M., Deaville, R., \& Rossiter, S. J. (2020). Phylogenomic resolution of the cetacean tree of life using target sequence capture. Systematic Biology, 69(3), 479-501.

Pyenson, N. D., Vélez-Juarbe, J., Gutstein, C. S., Little, H., Vigil, D., \& O’Dea, A. (2015). Isthminia panamensis, a new fossil inioid (Mammalia, Cetacea) from the Chagres Formation of Panama and the evolution of 'river dolphins' in the Americas. PeerJ, 3, e1227.

Swofford, D. L. 2003. PAUP*. Phylogenetic analysis using parsimony (*and other methods). Version 4.0 Sinauer Associates, Sunderland, MA. 